\title{
Resonance generation of sum harmonic in static electric field
}

\author{
E.F. Venger*, V.M. Griban, A.V. Melnichuk \\ *Institute of Semiconductor Physics, NAS of Ukraine, 45, prospekt Nauki, Kyiv, 252028, Ukraine \\ Phone: (38044) 26525 93; Fax: (38044) 26583 42; E-mail: mickle@semicond.kiev.ua \\ Mykola Gogol State Pedagogic University, 2 Kropyv'yans'kogo Str., Nizhin, 251200, Ukraine
}

\begin{abstract}
Using the group theory considerations, we investigate the nonlinear effect tensor (NET) for a crystal in a static electric field when a harmonic or the initial emission frequency approaches that of the excitonic absorption band. The dependence of additional terms in NET (resulting from the electric field applied) on the electric field components is found. Their form is shown to differ from that of usual NET. The results obtained are illustrated by considering the case of the $D_{2 d}$ crystal symmetry.
\end{abstract}

Keywords: sum harmonic, nonlinear effect tensor, group theory considerations.

Paper received 29.01.99; revised manuscript received 01.06.99; accepted for publication 11.10.99.

\section{Introduction}

The sum harmonic generation is among the nonlinear optical effects in crystals. It involves emission at a sum frequency, $\omega_{1}\left(\mathbf{k}_{1}\right)+\omega_{2}\left(\mathbf{k}_{2}\right)$, due to emission at frequencies $\omega_{1}\left(\mathbf{k}_{1}\right)$ and $\omega_{2}\left(\mathbf{k}_{2}\right)$. The well -known frequency doubling, i.e. the second harmonic generation, is a special case of the above effect.

When theoretically investigating the sum harmonic generation, it is convenient to use the nonlinear effect tensor (NET) $\alpha$. NET is a factor in the expression for the matrix element corresponding to an elementary act of the above process. The generation intensity is proportional to the NET modulus squared.

The NET $\alpha$ form was determined in the paper [1] using the polariton theory. The latter takes into account the retardation of interaction. This enables one to correctly analyze resonance phenomena that occur when an emission frequency approaches the excitonic absorption band frequency. The NET form in crystals of various symmetries was also investigated in the above paper.

Using the technique developed in the paper [1], one of the authors of the present paper has found the NET form for crystals in a static electric field $\mathbf{E}$ [2]. It was shown that the effect of the electric field results in appearance of an additional term $\alpha(\mathbf{E})$ in the NET $\alpha$. The form of this additional term, $\alpha(\mathbf{E})$, differs from that of $\alpha$. This means that, at some emission polarization, the harmonic generation is due to the field action only.

The tensor $\alpha(\mathbf{E})$ was found in the first order of the perturbation theory, so in general case the intensity of the sum harmonic generation due to it has to be much less than that due to the tensor $\alpha$. The tensor $\alpha(\mathbf{E})$, however, has resonance terms in denominators. This means that, when an emission frequency approaches the resonance, the effect of an electric field is to grow abruptly. Hence it follows that in the case of the resonance it is of both interest and importance to study the structure of the tensor $\alpha(\mathbf{E})$ and its transformation properties. The same is also true for the dependence of the tensor $\alpha(\mathbf{E})$ form on the symmetry type of the exciton state whose frequency is resonant. It is the above problems the present paper deals with.

\section{Results and discussion}

Let us give, first of all, the explicit form of the tensor $\alpha(\mathbf{E})$. According to the paper [2], it is a product of the vector $\mathbf{E}$ and a tensor $\beta$ of rank four:

$\alpha_{j j_{1} j_{2}}(\mathbf{E})=\sum_{i} E_{i} \beta_{j j_{1} j_{2} i}$

The tensor $\beta$ has 16 rather complicated components. For the following analysis it will be sufficient to give explicitly only one of them: 


\section{E.F. Venger et al.: Resonance generation of sum harmonic...}

$$
\begin{aligned}
& \beta_{j j_{1} j_{2} i}=-N \sum_{\mu_{1}, \mu_{2}, \mu^{\prime}, \mathbf{k}^{\prime}}\left\{\frac{\left(0\left|j_{2}\right| \mu^{\prime}, \mathbf{k}^{\prime}\right)\left(\mu^{\prime}, \mathbf{k}^{\prime}\left|p_{i}\right| \mu_{2}, \mathbf{k}_{2}\right)}{\left[E_{\mu_{2}}\left(\mathbf{k}_{2}\right)-E_{\mu^{\prime}}\left(\mathbf{k}^{\prime}\right)\right]} \times\right. \\
& \left.\times \frac{\left(\mu_{2}, \mathbf{k}_{2}\left|j_{1}\right| \mu_{1}, \mathbf{k}_{1}+\mathbf{k}_{2}\right)\left(\mu_{1}, \mathbf{k}_{1}+\mathbf{k}_{2}|j| 0\right)}{\left[E_{\mu_{2}}\left(\mathbf{k}_{2}\right)-\hbar \omega_{2}\left[E_{\mu_{1}}\left(\mathbf{k}_{1}+\mathbf{k}_{2}\right)-\hbar\left(\omega_{1}+\omega_{2}\right)\right]\right.}+\cdots\right\} .
\end{aligned}
$$

Here $N$ is the number of crystal unit cells; $\left.E_{\mu}(\mathbf{k}), \mid \mu, \mathbf{k}\right)$ are, respectively, the energy and wave function of a Coulomb exciton with wave vector $\mathbf{k}$ that belongs to the $\mu$-th band; $10)$ is the wave function for the ground state of the crystal; $j$, $j_{1}, j_{2}$ index the projections of polarization vectors for the harmonic and initial emission (they may take the values $x, y$, $z) ; p_{i}(i=x, y, z)$ is the $i$-th component of the electric dipole moment for a unit cell; $\left(\mu_{1}, \mathbf{k}_{1}\left|j_{n}\right| \mu_{2}, \mathbf{k}_{2}\right)$ is the matrix element of the operator $\frac{e}{m} I_{j_{n}}$ (where $e, m, I$ are, respectively, the electron charge, mass and momentum operator). The other terms in expression (2) differ from the above one by the place of the matrix element for the component $p_{i}$ and the differences (sums) in the denominators. The frequencies $\omega_{1}$ and $\omega_{2}$ can be considered as interchangeable, i.e., the right hand side of expression (2) is symmetric about interchanging the indices 1 and 2.

The form of the tensor $\alpha(\mathbf{E})$ depends on that of the tensor $\beta$. When determining the latter form, one has to keep in mind that the exciton states $\mid \mu, \mathbf{k})$ are classified according to the irreducible representations of the point group of crystal symmetry, while the expressions of the type

$$
\sum_{\mu} \frac{\mid \mu, \mathbf{k})(\mu, \mathbf{k} \mid}{E_{\mu}(\mathbf{k}) \pm \hbar \omega} .
$$

transform according to the entire symmetry representations [2]. So it follows from expression (2) that the tensor $\beta$ is isomorphic to $\left(j j_{1} j_{2} i\right)_{0}$ :

$$
\beta_{j j_{1} j_{2} i} \rightarrow\left(j j_{1} j_{2} i\right)_{0} .
$$

Here index 0 means that the product of coordinates, $j j_{1} j_{2} i$, is projected onto the entire symmetry representation of the point symmetry group. Basing on expression (3), the form of the tensor $\alpha(\mathbf{E})$ was determined in the paper [2] for crystals of various symmetries.

Now let us consider the form of the tensor $\alpha(\mathbf{E})$ when the sum frequency, $\omega_{1}+\omega_{2}$, approaches the excitonic band absorption frequency, $1 / \hbar \cdot E_{\mu}\left(\mathbf{k}_{1}+\mathbf{k}_{2}\right)$. (The case $\hbar\left(\omega_{1}+\right.$ $\left.\omega_{2}\right)=E_{\mu}\left(\mathbf{k}_{1}+\mathbf{k}_{2}\right)$ is, however, excluded. Such a two-photon absorption will be considered elsewhere.)

First of all, it should be noted that generally the terms with different $\mu_{1}$ make different contributions to different components of tensor $\beta$, depending on the $\left.\mid \mu_{1}, \mathbf{k}_{1}+\mathbf{k}_{2}\right)$ state symmetry. To demonstrate this, let us separate out those terms in expression (2) for which $\mu_{1}=\mu$ :

$$
\begin{gathered}
\beta_{j j_{1} j_{2} i}^{(\mu)}=-N \sum_{\mu_{2}, \mu^{\prime}, \mathbf{k}^{\prime}}\left\{\frac{\left(0\left|j_{2}\right| \mu^{\prime}, \mathbf{k}^{\prime}\right)\left(\mu^{\prime}, \mathbf{k}^{\prime}\left|p_{i}\right| \mu_{2}, \mathbf{k}_{2}\right)}{\left[E_{\mu_{2}}\left(\mathbf{k}_{2}\right)-E_{\mu^{\prime}}\left(\mathbf{k}^{\prime}\right)\right]} \times\right. \\
\left.\times \frac{\left(\mu_{2}, \mathbf{k}_{2}\left|j_{1}\right| \mu, \mathbf{k}_{1}+\mathbf{k}_{2}\right)\left(\mu, \mathbf{k}_{1}+\mathbf{k}_{2}|j| 0\right)}{\left[E_{\mu_{2}}\left(\mathbf{k}_{2}\right)-\hbar \omega_{2}\left[E_{\mu}\left(\mathbf{k}_{1}+\mathbf{k}_{2}\right)-\hbar\left(\omega_{1}+\omega_{2}\right)\right]\right.}+\cdots\right\}
\end{gathered}
$$

There is no summation over $\mu$ here, and hence the quantity

$$
\frac{\left.\mid \mu, \mathbf{k}_{1}+\mathbf{k}_{2}\right)\left(\mu, \mathbf{k}_{1}+\mathbf{k}_{2} \mid\right.}{\left[E_{\mu}\left(\mathbf{k}_{1}+\mathbf{k}_{2}\right)-\hbar\left(\omega_{1}+\omega_{2}\right)\right]}
$$

is not of full symmetry. This means that the term given in an explicit form in (4) is isomorphic not to $\left(j j_{1} j_{2} i\right)_{0}$ but to the product $\left(j_{2} i j_{1}\right) \mu(j) \mu$ where index $\mu$ signifies projection onto the irreducible representation to which the $\mid \mu_{1}, \mathbf{k}_{1}+$ $\mathbf{k}_{2}$ ) state belongs. An analysis made for the rest of terms in (4) (denoted by dots) shows that tensor $\beta^{(\mu)}$ is isomorphic to the following sum:

$\beta_{j j_{1} j_{2} i}^{(\mu)} \rightarrow(j)_{\mu}\left(j_{2} i j_{1}\right)_{\mu}+(i j)_{\mu}\left(j_{1} j_{2}\right)_{\mu}$.

After summing over $\mu$ one gets the relation (3) (as it was to be expected).

Identifying the index $\mu$ with designation of the corresponding irreducible representation, one can determine the factors in the right-hand side of expression (5) and obtain first the tensor $\beta^{(\mu)}$ and then the tensor $\alpha^{(\mu)}(\boldsymbol{E})$ :

$$
\alpha_{j j_{1} j_{2}}^{(\mu)}(\mathbf{E})=\sum_{i} E_{i} \beta_{j j_{1} j_{2} i}^{(\mu)}
$$

After summing the corresponding tensor $\alpha^{(\mu)}(\mathbf{E})$ components over all the irreducible representations, one can get the tensor $\alpha(\mathbf{E})$. Its form is to be the same as that following from expression (3).

Now let us return to the resonance conditions when $\hbar\left(\omega_{1}+\omega_{2}\right) \rightarrow E_{\mu}\left(\mathbf{k}_{1}+\mathbf{k}_{2}\right)$. In this case the terms in $\beta^{(\mu)}$ with $\mu=\mu_{1}$ will prevail. As a result, the components $\alpha^{(\mu)}(\mathbf{E})$ of the tensor $\alpha(\mathbf{E})$ will prevail. Thus under resonance conditions one can write down:

$$
\begin{aligned}
& \beta=\sum_{\mu_{1}} \beta^{\left(\mu_{1}\right)}=\sum_{\mu_{1} \neq \mu} \beta^{\left(\mu_{1}\right)}+\beta^{(\mu)}, \\
& \alpha(\mathbf{E})=\sum_{\mu_{1}} \alpha^{\left(\mu_{1}\right)}(\mathbf{E})=\sum_{\mu_{1} \neq \mu} \alpha^{\left(\mu_{1}\right)}(\mathbf{E})+\alpha^{(\mu)}(\mathbf{E}) .
\end{aligned}
$$

According to the above considerations, the nonresonance components may be neglected and therefore $\alpha(\mathbf{E}) \approx \alpha^{(\mu)}(\mathbf{E})$. This means that under resonance conditions the tensor $\alpha(\mathbf{E})$ retains its form; $\alpha^{(\mu)}(\mathbf{E})$, however, become the governing components. Similar reasoning was used in paper [3] to analyze the Raman scattering tensor under resonance conditions. 


\section{E.F. Venger et al.: Resonance generation of sum harmonic...}

To illustrate the above results, let us consider a crystal of $D_{2 d}$ symmetry. For it the usual NET has the following non-zero components: $\alpha_{x y z}=\alpha_{x z y}=\alpha_{y x z}=\alpha_{y z x}, \alpha_{z x y=} \alpha_{z y x}$. In a static electric field the tensor $\alpha(\mathbf{E})$ appears whose form follows from expression (3). It is given in Table 2 - see the row designated by $\sum_{\mu}$.

Let us determine the tensor $\alpha^{(\mu)}(\mathbf{E})$. The $D_{2 d}$ group has four one-dimensional ( $1 D$ ) irreducible representations, $A_{1}$, $A_{2}, B_{1}, B_{2}$, and a $2 D$ irreducible representation, $E$, with two rows, $E^{(x)}$ and $E^{(y)}$, after which the coordinates $x, y$ transform.

Using expression (5), one can find the form of the tensor $\beta^{(\mu)}$. To this end one has to determine the irreducible representations of the $D_{2 d}$ group after which the coordinates $x, y, z$, as well as their double and triple products, transform. The corresponding technique is discussed in the papers $[3,4]$. The results of our calculations are given in Table 1 .

Let us consider the case $\mu=A_{1}$, i.e. the wave function of the intermediate state, $\left.\mid \mu_{1}, \mathbf{k}_{1}+\mathbf{k}_{2}\right)$, corresponds to the irreducible representation $A_{1}$. One can conclude from Table 1 that projections of coordinates $x, y, z$ onto the representation $A_{1}$ are zero. Therefore, the first term in the right-hand side of expression (5) drops out. For the second term the non-zero projections of double products are $\left(x_{1} x_{2}\right)_{A 1}=\left(y_{1} y_{2}\right)_{A 1}$ and $\left(z_{1} z_{2}\right)_{A 1}$. Thus, according to expression (5), the non-zero components of the tensor $\beta(\mu)$ are the following:

$\beta_{x x x x}^{\left(A_{1}\right)}=\beta_{x x y y}^{\left(A_{1}\right)}=\beta_{y y x x}^{\left(A_{1}\right)}=a_{1}, \quad \beta_{z z z z}^{\left(A_{1}\right)}=a_{2}$,

$\beta_{x x z z}^{\left(A_{1}\right)}=\beta_{y y z z}^{\left(A_{1}\right)}=a_{3}, \quad \beta_{z z x x}^{\left(A_{1}\right)}=\beta_{z z y y}^{\left(A_{1}\right)}=a_{4}$.

Thereafter, using (6), one can determine the form of the tensor $\alpha^{\left(A_{1}\right)}(\mathbf{E})$ :

$\alpha_{x x x}^{\left(A_{1}\right)}(\mathbf{E})=a_{1} E_{x}, \alpha_{y y y}^{\left(A_{1}\right)}(\mathbf{E})=a_{1} E_{y}, \alpha_{z z z}^{\left(A_{1}\right)}(\mathbf{E})=a_{2} E_{z}$,

$\alpha_{y x x}^{\left(A_{1}\right)}(\mathbf{E})=a_{1} E_{y}, \alpha_{x y y}^{\left(A_{1}\right)}(\mathbf{E})=a_{1} E_{x}, \alpha_{z x x}^{\left(A_{1}\right)}(\mathbf{E})=a_{3} E_{z}$,

$\alpha_{z y y}^{\left(A_{1}\right)}(\mathbf{E})=a_{3} E_{z}, \alpha_{x z z}^{\left(A_{1}\right)}(\mathbf{E})=a_{4} E_{x}, \alpha_{y z z}^{\left(A_{1}\right)}(\mathbf{E})=a_{4} E_{y}$.
All the other components are zero.

The same procedure can be used to find the tensor $\alpha^{(\mu)}(\mathbf{E})$ components for other irreducible representations of the $D_{2 d}$ group. The corresponding results are given in Table 2. (There the numbers $1,2, \ldots$ are used instead of $a_{1}, a_{2}, \ldots$, and the relations between components correspond to the only representation.)

After performing summation of the corresponding components over all the irreducible representations, one can obtain the tensor form in the case when there is no resonance (it is given in the row designated with $\sum_{\mu}$ ).

Now let us consider the case when the frequency $\omega_{2}$ approaches the frequency of the excitonic absorption band. One can show that an isomorphism at the initial emission frequency follows from expression (2):

$$
\beta_{j j_{1} j_{2} i}^{(\mu)} \rightarrow\left(j_{2} j\right)_{\mu}\left(j j_{1}\right)_{\mu}+\left(j_{2}\right)_{\mu}\left(j i j_{1}\right)_{\mu}
$$

If $\mu=A_{2}$, then, basing on expressions (9) and (1) and Table 1 , we get:

$$
\begin{array}{ll}
\alpha_{x y x}^{\left(A_{2}\right)}(\mathbf{E})=a_{1} E_{y}, & \alpha_{y x x}^{\left(A_{2}\right)}(\mathbf{E})=-a_{1} E_{y}, \\
\alpha_{y x y}^{\left(A_{2}\right)}(\mathbf{E})=a_{1} E_{x}, & \alpha_{x y y}^{\left(A_{2}\right)}(\mathbf{E})=-a_{1} E_{x} .
\end{array}
$$

All the other components are zero.

The form of the tensor $\alpha^{(\mu)}(\mathbf{E})$ in this case is given in Table 3. This tensor is non-symmetric in respect to the indices $j_{1}, j_{2}$, because there is no symmetry now between the frequencies $\omega_{1}$ and $\omega_{2}: \omega_{2}$ is the resonance frequency, while $\omega_{1}$ is not.

When the resonance occurs at a frequency $\omega_{1}$, then one has to interchange the components $\alpha_{j j_{1} j_{2}}^{(\mu)}$ and $\alpha_{j j_{2} j_{1}}^{(\mu)}$ in Table 3. If frequency is doubled $\left(\omega_{1}=\omega_{2}=\omega\right)$, then one

\begin{tabular}{|c|c|c|c|c|c|c|c|c|c|c|}
\hline$\mu$ & \multicolumn{10}{|c|}{ Coordinates and their products } \\
\hline$A_{1}$ & \multicolumn{2}{|c|}{$x_{1} x_{2}+y_{1} y_{2}$} & \multicolumn{2}{|c|}{$z_{1} z_{2}$} & \multicolumn{2}{|c|}{$x_{1} y_{2} z_{3}+y_{1} x_{2} z_{3}$} & \multicolumn{2}{|c|}{$x_{1} z_{2} y_{3}+y_{1} z_{2} x_{3}$} & \multicolumn{2}{|c|}{$z_{1} x_{2} y_{3}+z_{1} y_{2} x_{3}$} \\
\hline$A_{2}$ & \multicolumn{2}{|c|}{$x_{1} y_{2}-y_{1} x_{2}$} & \multicolumn{2}{|c|}{$x_{1} x_{2} z_{3}-y_{1} y_{2} z_{3}$} & \multicolumn{2}{|c|}{$x_{1} z_{2} x_{3}-y_{1} z_{2} y_{3}$} & \multicolumn{2}{|c|}{$z_{1} x_{2} x_{3}-z_{1} y_{2} y_{3}$} & & \\
\hline$B_{1}$ & \multicolumn{2}{|c|}{$x_{1} x_{2}-y_{1} y_{2}$} & \multicolumn{2}{|c|}{$x_{1} y_{2} z_{3}-y_{1} x_{2} z_{3}$} & \multicolumn{2}{|c|}{$x_{1} z_{2} y_{3}-y_{1} z_{2} x_{3}$} & \multicolumn{2}{|c|}{$z_{1} x_{2} y_{3}-z_{1} y_{2} x_{3}$} & & \\
\hline$B_{2}$ & \multicolumn{2}{|c|}{$z$} & \multicolumn{2}{|c|}{$z_{1} z_{2} z_{3}$} & \multicolumn{2}{|c|}{$x_{1} x_{2} z_{3}+y_{1} y_{2} z_{3}$} & \multicolumn{2}{|c|}{$x_{1} z_{2} x_{3}+y_{1} z_{2} y_{3}$} & \multicolumn{2}{|c|}{$z_{1} x_{2} x_{3}+z_{1} y_{2} y_{3}$} \\
\hline$E^{(x)}$ & $x$ & $y_{1} z_{2}$ & $z_{1} y_{2}$ & $x_{1} x_{2} x_{3}$ & $y_{1} y_{2} x_{3}$ & $y_{1} x_{2} y_{3}$ & $x_{1} y_{2} y_{3}$ & $z_{1} z_{2} x_{3}$ & $z_{1} x_{2} z_{3}$ & $x_{1} z_{2} z_{3}$ \\
\hline$E^{(y)}$ & $y$ & $x_{1} z_{2}$ & $z_{1} x_{2}$ & $y_{1} y_{2} y_{3}$ & $x_{1} x_{2} y_{3}$ & $x_{1} y_{2} x_{3}$ & $y_{1} x_{2} x_{3}$ & $z_{1} z_{2} y_{3}$ & $z_{1} y_{2} z_{3}$ & $y_{1} z_{2} z_{3}$ \\
\hline
\end{tabular}
has to take $\alpha_{j j_{1} j_{2}}^{(\mu)}=\alpha_{j j_{2} j_{1}}^{(\mu)}$.

Table 1. Coordinates and their products that transform according to the irreducible representations of the $D_{2 d}$ group. 
E.F. Venger et al.: Resonance generation of sum harmonic...

Table 2. Form of the tensor $\alpha^{(\mu)}(\mathbf{E})$ for the resonance frequency $\omega_{1}+\omega_{2}$.

\begin{tabular}{|c|c|c|c|c|c|c|c|c|c|}
\hline \multirow{4}{*}{$\mu$} & \multicolumn{9}{|c|}{$j j_{1} j_{2}$} \\
\hline & $x x x$ & yyy & $z z z$ & $x x z$ & $x z x$ & $z x x$ & $z z y$ & $z y z$ & $y z z$ \\
\hline & $x x y$ & $x y x$ & $y x x$ & $y y z$ & $y z y$ & zyy & $x y z$ & $x z y$ & $z x y$ \\
\hline & $y y x$ & $y x y$ & xyy & $z z x$ & $z x z$ & $x z z$ & $y x z$ & $y z x$ & $z y x$ \\
\hline \multirow{3}{*}{$A_{1}$} & $1 E_{x}$ & $1 E_{y}$ & $2 E_{z}$ & 0 & 0 & $3 E_{z}$ & 0 & 0 & $4 E_{z}$ \\
\hline & 0 & 0 & $1 E_{y}$ & 0 & 0 & $3 E_{z}$ & 0 & 0 & 0 \\
\hline & 0 & 0 & $1 E_{x}$ & 0 & 0 & $4 E_{x}$ & 0 & 0 & 0 \\
\hline \multirow{3}{*}{$A_{2}$} & 0 & 0 & $4 E_{y}$ & 0 & 0 & 0 & 0 & 0 & 0 \\
\hline & 0 & 0 & 0 & 0 & 0 & 0 & 0 & 0 & 0 \\
\hline & 0 & 0 & 0 & 0 & 0 & 0 & 0 & 0 & 0 \\
\hline \multirow{3}{*}{$B_{1}$} & $1 E_{x}$ & $1 E_{y}$ & 0 & 0 & 0 & 0 & 0 & 0 & 0 \\
\hline & 0 & 0 & $-1 E_{y}$ & 0 & 0 & 0 & 0 & 0 & 0 \\
\hline & 0 & 0 & $-1 E_{x}$ & 0 & 0 & 0 & 0 & 0 & 0 \\
\hline \multirow{3}{*}{$B_{2}$} & 0 & 0 & $1 E_{z}$ & 0 & 0 & $3 E_{z}$ & $4 E_{y}$ & $4 E_{y}$ & 0 \\
\hline & $2 E_{y}$ & $2 E_{y}$ & 0 & 0 & 0 & $3 E_{z}$ & 0 & 0 & 0 \\
\hline & $2 E_{x}$ & $2 E_{x}$ & 0 & $4 E_{x}$ & $4 E_{x}$ & 0 & 0 & 0 & 0 \\
\hline \multirow{3}{*}{$E^{(x)}$} & $1 E_{x}$ & 0 & 0 & $4 E_{z}$ & $4 E_{z}$ & 0 & $7 E_{y}$ & $7 E_{y}$ & 0 \\
\hline & $2 E_{y}$ & $2 E_{y}$ & 0 & $5 E_{z}$ & $5 E_{z}$ & 0 & 0 & 0 & 0 \\
\hline & 0 & 0 & $3 E_{x}$ & 0 & 0 & $6 E_{x}$ & 0 & 0 & 0 \\
\hline \multirow{3}{*}{$E^{(y)}$} & 0 & $1 E_{y}$ & 0 & $4 E_{z}$ & $4 E_{z}$ & 0 & 0 & 0 & $7 E_{y}$ \\
\hline & 0 & 0 & $2 E_{y}$ & $5 E_{z}$ & $5 E_{z}$ & 0 & 0 & 0 & 0 \\
\hline & $3 E_{x}$ & $3 E_{x}$ & 0 & $6 E_{x}$ & $6 E_{x}$ & 0 & 0 & 0 & 0 \\
\hline & $1 E_{x}$ & $2 E_{y}$ & $3 E_{z}$ & $8 E_{z}$ & $8 E_{z}$ & $9 E_{z}$ & $14 E_{y}$ & $14 E_{y}$ & $15 E_{y}$ \\
\hline$\sum$ & $4 E_{y}$ & $4 E_{y}$ & $5 E_{y}$ & $10 E_{z}$ & $10 E_{z}$ & $11 E_{z}$ & 0 & 0 & 0 \\
\hline$L_{\mu}$ & $6 E_{x}$ & $6 E_{x}$ & $7 E_{x}$ & $12 E_{x}$ & $12 E_{x}$ & $13 E_{x}$ & 0 & 0 & 0 \\
\hline
\end{tabular}

Table 3. Form of the tensor $\alpha^{(\mu)}(\mathrm{E})$ for the resonance frequency $\omega_{2}$.

\begin{tabular}{|c|c|c|c|c|c|c|c|c|c|}
\hline \multirow[t]{4}{*}{$\mu$} & \multicolumn{9}{|c|}{$j j_{1} j_{2}$} \\
\hline & $x x x$ & yyy & $z z z$ & $x x z$ & $x z x$ & $z x x$ & $z z y$ & $z y z$ & $y z z$ \\
\hline & $x x y$ & $x y x$ & $y x x$ & $y y z$ & $y z y$ & zyy & $x y z$ & $x z y$ & $z x y$ \\
\hline & $y y x$ & $y x y$ & xyy & $z z x$ & $z x z$ & $x z z$ & $y x z$ & $y z x$ & $z y x$ \\
\hline \multirow{3}{*}{$A_{1}$} & $1 E_{x}$ & $1 E_{y}$ & $2 E_{z}$ & 0 & $3 E_{z}$ & 0 & 0 & 0 & 0 \\
\hline & 0 & $1 E_{y}$ & 0 & 0 & $3 E_{z}$ & 0 & 0 & 0 & 0 \\
\hline & 0 & $1 E_{x}$ & 0 & 0 & $4 E_{x}$ & 0 & 0 & 0 & 0 \\
\hline \multirow{3}{*}{$A_{2}$} & 0 & 0 & 0 & 0 & 0 & 0 & 0 & 0 & 0 \\
\hline & $1 E_{y}$ & 0 & $-1 E_{y}$ & 0 & 0 & 0 & 0 & 0 & 0 \\
\hline & $1 E_{x}$ & 0 & $-1 E_{x}$ & 0 & 0 & 0 & 0 & 0 & 0 \\
\hline \multirow{3}{*}{$B_{1}$} & $1 E_{x}$ & $1 E_{y}$ & 0 & 0 & 0 & 0 & 0 & 0 & 0 \\
\hline & 0 & $-1 E_{y}$ & 0 & 0 & 0 & 0 & 0 & 0 & 0 \\
\hline & 0 & $-1 E_{x}$ & 0 & 0 & 0 & 0 & 0 & 0 & 0 \\
\hline \multirow{3}{*}{$B_{2}$} & 0 & 0 & $1 E_{z}$ & 0 & $3 E_{z}$ & 0 & $4 E_{y}$ & 0 & $5 E_{y}$ \\
\hline & $2 E_{y}$ & 0 & 0 & 0 & $3 E_{z}$ & 0 & 0 & 0 & 0 \\
\hline & $2 E_{x}$ & 0 & 0 & $4 E_{x}$ & 0 & $5 E_{x}$ & 0 & 0 & 0 \\
\hline \multirow{3}{*}{$E^{(x)}$} & $1 E_{x}$ & 0 & 0 & $5 E_{z}$ & 0 & $6 E_{z}$ & $10 E_{y}$ & 0 & $11 E_{y}$ \\
\hline & $2 E_{y}$ & 0 & $3 E_{y}$ & $7 E_{z}$ & 0 & $8 E_{z}$ & 0 & 0 & 0 \\
\hline & 0 & $4 E_{x}$ & 0 & 0 & $9 E_{x}$ & 0 & 0 & 0 & 0 \\
\hline \multirow{3}{*}{$E^{(y)}$} & 0 & $1 E_{y}$ & 0 & $5 E_{z}$ & 0 & $6 E_{z}$ & 0 & $11 E_{y}$ & 0 \\
\hline & 0 & $2 E_{y}$ & 0 & $7 E_{z}$ & 0 & $8 E_{z}$ & 0 & 0 & 0 \\
\hline & $3 E_{x}$ & 0 & $4 E_{x}$ & $9 E_{x}$ & 0 & $10 E_{x}$ & 0 & 0 & 0 \\
\hline
\end{tabular}




\section{E.F. Venger et al.: Resonance generation of sum harmonic...}

\section{Conclusions}

From the above results it follows that, under the resonance generation of the sum harmonic in a static electric field, the nonlinear effect tensor involves a term $\alpha^{(\mu)}(\mathbf{E})$ that depends on both the field $\mathbf{E}$ and the symmetry type of the exciton state whose frequency is the resonance one. The form of the tensor $\alpha^{(\mu)}(\mathbf{E})$ differs from that of the non-resonance nonlinear effect tensor. Besides, it is different at the frequencies of the harmonic and initial emission.

\section{References}

1. L.N. Ovander, Nonlinear optical effects in crystals // Uspekhi Fiz. Nauk 86 (1), pp. 1-39 (1965) (in Russian).

2. V.M. Griban, On sum harmonic generation in crystals with inversion center (in Russian) // Izv. Vuzov SSSR. Fizika N 6, pp.107-110 (1969).

3. L.D. Landau, E.M. Lifshits, Quantum Mechanics, Nauka, Moscow, p.443 (1989) (in Russian)

4. M.I. Petrashen, E.D. Trifonov, Group Theory Applications in Quantum Mechanics, Nauka, Moscow (1967) (in Russian). 